\title{
GLOBAL EXISTENCE OF SOLUTIONS OF PARABOLIC PROBLEMS WITH NONLINEAR BOUNDARY CONDITIONS
}

\author{
PAVOL QUITTNER \\ Institute of Applied Mathematics, Comenius University \\ Mlynská dolina, 84215 Bratislava, Slovakia \\ E-mail: quittner@fmph.uniba.sk
}

In [1], H. Amann derived an a priori bound for solutions of parabolic problems with nonlinear boundary conditions in the Sobolev space $W_{p}^{s}\left(\Omega, \mathbb{R}^{N}\right)\left(s \geq 1, p>n, \Omega \subset \mathbb{R}^{n}\right.$ bounded). The result ([1, Theorem 15.2]) is based on the assumptions of an apriori estimate for the solutions in some weaker norm (in $W_{p_{0}}^{s_{0}}\left(\Omega, \mathbb{R}^{N}\right), s>s_{0}, p_{0} \geq 1$ ) and of suitable growth conditions for the local nonlinearities arising in the problem. However, the proof of this result contains some discrepancies (the choice of $r$ in the proof does not match the assumptions in [1, Lemma 15.1]) and the result itself is not correct in the case $n=1$ : the growth of the function $g$ arising in the boundary condition has to be controlled by the power $1+p_{0} /\left(n-s_{0} p_{0}\right)$ also in this case. The aim of this paper is to give a correct proof of a modification of the result mentioned above and to show that the growth assumption is optimal for $n=1$.

The idea of our proof is the same as that in [1]. For the sake of simplicity we consider only the special case $s_{0}=0$. On the other hand, unlike [1] we do not assume $p>n$. We consider the problem

$$
\begin{cases}u_{t}+\mathcal{A} u=f(x, t, u, \nabla u) & \text { in } \Omega \times(0, T), \\ \mathcal{B} u=g(x, t, u) & \text { on } \partial \Omega \times(0, T), \\ u(x, 0)=u_{0}(x), & x \in \bar{\Omega},\end{cases}
$$

where $0<T \leq \infty, \Omega$ is a bounded domain in $\mathbb{R}^{n}$ of class $C^{2}, u: \Omega \times[0, T) \rightarrow \mathbb{R}^{N}$, $\mathcal{A} u=\left(-\Delta u_{1}, \ldots,-\Delta u_{N}\right), \mathcal{B} u=\partial u / \partial n$ is the derivative with respect to the outer normal on the boundary $\partial \Omega$ (the generalization to more complicated, non-autonomous

1991 Mathematics Subject Classification: Primary 35K60; Secondary 35B35.

The paper is in final form and no version of it will be published elsewhere. 
operators $\mathcal{A}, \mathcal{B}$ as in [1] is straightforward), $f, g$ are $C^{1}$ functions with

$$
\begin{aligned}
\left|\partial_{t} f(x, t, \xi, \eta)\right| & \leq C\left(1+|\xi|^{2 \nu_{1}+1}+|\eta|^{\nu_{2}+1}\right) \\
\left|\partial_{\xi} f(x, t, \xi, \eta)\right| & \leq C\left(1+|\xi|^{2 \nu_{1}}+|\eta|^{\nu_{2}+\min \left(1, \nu_{2}\right)}\right), \\
\left|\partial_{\eta} f(x, t, \xi, \eta)\right| & \leq C\left(1+|\xi|^{\nu_{1}}+|\eta|^{\nu_{2}}\right) \\
\left|\partial_{t} g(x, t, \xi)\right| & \leq C\left(1+|\xi|^{\nu_{1}+1}\right) \\
\left|\partial_{\xi} g(x, t, \xi)\right| & \leq C\left(1+|\xi|^{\nu_{1}}\right)
\end{aligned}
$$

for some $\nu_{1}<p /(n-p)$ (if $\left.p<n\right), \nu_{2}<p / n$ and $p>1$ (the assumptions concerning the smoothness of $f, g$ in $t$ and $x$ can be relaxed; see e.g. [1, p. 255] for sufficient assumptions in the case $p>n$ ).

If $u_{0} \in W_{p}^{s}\left(\Omega, \mathbb{R}^{N}\right), s \in[1,1+1 / p)$, then the theory developed in [1] guarantees the existence of a unique maximal solution of $(\mathrm{P})$ in $W_{p}^{s}\left(\Omega, \mathbb{R}^{N}\right)$. Moreover, $u(t) \in W_{p}^{s+\varepsilon}\left(\Omega, \mathbb{R}^{N}\right)$ for some $\varepsilon>0$ and any $t>0$ and a simple bootstrap argument together with standard imbedding theorems show that $u(t) \in W_{\tilde{p}}^{\tilde{s}}\left(\Omega, \mathbb{R}^{N}\right)$ for any $\tilde{p} \geq p, \tilde{s}<1+1 / \tilde{p}$ and $t>0$. The solution fulfils a variation-of-constants formula of the form

$$
u(t)=e^{-A t} u_{0}+\int_{0}^{t} e^{-A(t-s)} F(s, u(s)) d s,
$$

where $A$ is an operator associated with the differential operators $\mathcal{A}, \mathcal{B}$ and $F$ is a map induced by the nonlinear functions $f, g$ (see $[1$, p. 244] for details). The results of $[1$, Section 12] imply also that this solution is global if the map $F$ fulfils an estimate of the type

$$
\|F(t, u(t))\|_{W_{\mathcal{B}}^{s^{\prime} / 2-1}} \leq c(t)\left(1+\|u(t)\|_{W_{\mathcal{B}}^{s / 2}}^{\varepsilon}\right)
$$

for some $\varepsilon<1, s<s^{\prime}<1+1 / p$ and a nondecreasing function $c: \mathbb{R}^{+} \rightarrow \mathbb{R}^{+}$(where $W_{\mathcal{B}}^{s / 2}=W_{p}^{s}\left(\Omega, \mathbb{R}^{N}\right)$ and the extrapolation space $W_{\mathcal{B}}^{s^{\prime} / 2-1}$ can be viewed as the dual of the space $W_{q}^{2-s^{\prime}}\left(\Omega, \mathbb{R}^{N}\right)$ with $1 / p+1 / q=1$; see $\left.[1]\right)$. Moreover, if $T=\infty$ and $c: \mathbb{R}^{+} \rightarrow \mathbb{R}^{+}$ is bounded then $u:[0, T) \rightarrow W_{p}^{s}\left(\Omega, \mathbb{R}^{N}\right)$ is bounded.

Our main result is the following modification of $\left[1\right.$, Theorem 15.2]. By $\|\cdot\|_{s, p}$ or $\|\cdot\|_{p}$ we denote the norm in $W_{p}^{s}\left(\Omega, \mathbb{R}^{N}\right)$ or $L_{p}\left(\Omega, \mathbb{R}^{N}\right)$, respectively.

Theorem. Let $p_{0} \geq 1, p>\max \left(1, p_{0}(n-1) /\left(p_{0}+n\right)\right), \hat{\lambda}_{1}<1+1 / p$,

$$
\begin{aligned}
1 & \leq \hat{\lambda}_{j}<1+\frac{p_{0}(2-j)}{n+j p_{0}}, \quad j=0,1, \\
1 & \leq \hat{\lambda}<1+\frac{p_{0}}{n} \\
|f(x, t, \xi, \eta)| & \leq C\left(1+|\xi|^{\hat{\lambda}_{0}}+|\eta|^{\hat{\lambda}_{1}}\right), \\
|g(y, t, \xi)| & \leq C\left(1+|\xi|^{\hat{\lambda}}\right)
\end{aligned}
$$

for $x \in \bar{\Omega}, y \in \partial \Omega, t \in[0, T)$ and $(\xi, \eta) \in \mathbb{R}^{N} \times \mathbb{R}^{N n}$. Let $u_{0} \in W_{p}^{1}\left(\Omega, \mathbb{R}^{N}\right)$ and let $u$ be the corresponding maximal solution of $(P)$ with the maximal existence time $T_{\max } \leq T$. Let $c: \mathbb{R}^{+} \rightarrow \mathbb{R}^{+}$be a nondecreasing function and let $\|u(t)\|_{p_{0}} \leq c(t)$ for any $t \in\left[0, T_{\max }\right)$. Then $T_{\max }=T$ and $\sup _{t \in\left[t_{1}, t_{2}\right)}\|u(t)\|_{s, p}<\infty$ for any $s<1+1 / p, t_{1}>0$ and $t_{2} \leq T$, $t_{2}<\infty\left(\right.$ or $t_{2}=\infty$ if $T=\infty$ and $c: \mathbb{R}^{+} \rightarrow \mathbb{R}^{+}$is bounded $)$. 
Remark 1. The assumption $\hat{\lambda}_{1}<1+1 / p$ seems to be of technical nature: it is due to the fact that we work in the space $W_{p}^{s}\left(\Omega, \mathbb{R}^{N}\right)$ with $s<1+1 / p$ which is required by the nonlinear boundary conditions. If we consider e.g. homogeneous Dirichlet boundary conditions then one can use the variation-of-constants formula and corresponding estimates in the space $W_{p}^{s}\left(\Omega, \mathbb{R}^{N}\right)$ for any $s<2$ and the assumption $\hat{\lambda}_{1}<1+1 / p$ becomes unnecessary (cf. also [2, Theorem 5.3]).

The proof of the Theorem is based on the following three lemmas.

Lemma 1. Let $p_{0}, \lambda, r \geq 1, \lambda r>1, p>1, s, \sigma \in[0,2], s>0$ and

$$
1+p_{0}(1 / r-1 / p)<\lambda<1+p_{0} \frac{s-\sigma+n(1 / r-1 / p)}{n+\sigma p_{0}} .
$$

Then there exists $\varepsilon \in(0,1)$ such that

$$
\|u\|_{\sigma, r \lambda}^{\lambda} \leq C\|u\|_{p_{0}}^{\lambda-\varepsilon}\|u\|_{s, p}^{\varepsilon} \quad \text { for any } u \in W_{p}^{s}\left(\Omega, \mathbb{R}^{N}\right) \cap L_{p_{0}}\left(\Omega, \mathbb{R}^{N}\right) .
$$

Proof. The proof follows from [1, Lemma 15.1] by choosing $\varepsilon$ sufficiently close to $1, s_{0}=0$ and observing that the assumption $r \geq p \geq p_{0}$ in [1] can be relaxed to the assumption $1 /(\lambda r)<(1-1 / \lambda) / p_{0}+(1 / \lambda) / p$ (cf. [2, Proposition 4.1]) which is equivalent to $\lambda>1+p_{0}(1 / r-1 / p)$.

LEMMA 2. Let $p_{0} \geq 1, p>\max \left(1, p_{0}(n-1) /\left(p_{0}+n\right)\right), 1 \leq \hat{\lambda}<1+p_{0} / n$. If $s \in[1,1+1 / p)$ is sufficiently close to $1+1 / p$ then there exist $r \geq 1$ and $\lambda \geq \hat{\lambda}$ such that $r>p(n-1) /(n-p(s-1)), r \lambda<p(n-1) /(n-s p)($ if $n>s p)$ and $(\mathrm{A})$ is fulfilled with $\sigma=1 /(\lambda r)$.

Proof. If $n>1$ choose $s \in[1,1+1 / p)$ such that $s>\max (2-n+n / p, 1 / n+1 / p)$. Then $\tilde{r}:=p(n-1) /(n-p(s-1))>1$. Choose $r>\tilde{r}$ such that $r\left(1+p_{0} / n\right)<p(n-1) /(n-s p)$ (if $n>s p$ ) and $\lambda_{\max }(r)>\max \left(\hat{\lambda}, \lambda_{\min }(r)\right)$, where

$$
\lambda_{\min }(r):=1+p_{0}(1 / r-1 / p) \quad \text { and } \quad \lambda_{\max }(r):=\lambda_{\min }(r)+\left(p_{0} / n\right)(s-1 / r) .
$$

This is possible since $\tilde{r}\left(1+p_{0} / n\right)<p(n-1) /(n-s p)$ (if $\left.n>s p\right)$ and $\lambda_{\max }(\tilde{r})=1+p_{0} / n>$ $\lambda_{\min }(\tilde{r})$. If $n=1$ and $r>1$ is arbitrary then $\lambda_{\max }(r)=1+p_{0}(s-1 / p)>\max \left(\hat{\lambda}, \lambda_{\min }(r)\right)$ if $s$ is sufficiently close to $1+1 / p$.

Now for any $n \geq 1$ choose $\lambda \in\left(\max \left(\hat{\lambda}, \lambda_{\min }(r)\right), \lambda_{\max }(r)\right)$. This choice guarantees (A) with $\sigma=1 /(\lambda r)$ since the second inequality in $(\mathrm{A})$ is equivalent to $\lambda<\lambda_{\max }(r)$ in this case.

LEMma 3. Let $p_{0} \geq 1, p>\max \left(1, p_{0}(n-1) /\left(p_{0}+n\right)\right), 1 \leq \hat{\lambda}_{0}<1+2 p_{0} / n, s \in$ $[1,1+1 / p), s^{\prime} \in(s, 1+1 / p)$. Put $r=p n /\left(n+\left(2-s^{\prime}\right) p\right)$ if $n>1, r=1$ if $n=1$. If $s \in[1,1+1 / p)$ is sufficiently close to $1+1 / p$ then there exists $\lambda_{0}>\hat{\lambda}_{0}$ such that $r \lambda_{0}<p n /(n-s p)($ if $n>s p)$ and $(\mathrm{A})$ is fulfilled with $\sigma=0$ and $\lambda$ replaced by $\lambda_{0}$. If , moreover, $1 \leq \hat{\lambda}_{1}<1+\min \left(p_{0} /\left(n+p_{0}\right), 1 / p\right)$ then there exist $R \geq r$ and $\lambda_{1}>\hat{\lambda}_{1}$ such that $R \lambda_{1}<p n /(n-(s-1) p)$ and $(\mathrm{A})$ is fulfilled with $\sigma=1, r$ replaced by $R$ and $\lambda$ replaced by $\lambda_{1}$.

Proof. Denote $\lambda_{\min }=1+p_{0}(1 / r-1 / p), \lambda_{\max }=\lambda_{\min }+\left(p_{0} / n\right) s$. Then (A) with 
$\sigma=0$ is equivalent to $\lambda_{\min }<\lambda<\lambda_{\max }$. It is easy to see that

$$
\begin{aligned}
1+\frac{2 p_{0}}{n}>\lambda_{\max }=1+\frac{2 p_{0}}{n}-p_{0} \frac{s^{\prime}-s}{n}>\max \left(\hat{\lambda}_{0}, \lambda_{\min }\right) & \text { if } n>1, \\
1+\frac{2 p_{0}}{n}>\lambda_{\max }=1+2 p_{0}-p_{0}(1+1 / p-s)>\max \left(\hat{\lambda}_{0}, \lambda_{\min }\right) & \text { if } n=1
\end{aligned}
$$

provided $s$ is sufficiently close to $1+1 / p$. Moreover, $r\left(1+2 p_{0} / n\right)<p n /(n-s p)$ if $n>s p$ and $s$ is close to $1+1 / p$ due to our assumption $p>p_{0}(n-1) /\left(p_{0}+n\right)$. Hence, it is sufficient to choose $\lambda_{0} \in\left(\max \left(\hat{\lambda}_{0}, \lambda_{\min }\right), \lambda_{\max }\right)$.

Now let $1 \leq \hat{\lambda}_{1}<1+\min \left(p_{0} /\left(n+p_{0}\right), 1 / p\right)$.

If $n>p_{0}(p-1)$ (i.e. $\left.p_{0} /\left(n+p_{0}\right)<1 / p\right)$, put $R=r$,

$$
\Lambda_{\max }=1+p_{0} \frac{s-1+n(1 / R-1 / p)}{n+p_{0}}, \quad \Lambda_{\min }=1+p_{0}(1 / R-1 / p) .
$$

Then

$$
\begin{array}{lll}
\Lambda_{\max }=1+\frac{p_{0}}{n+p_{0}}\left(1-\left(s^{\prime}-s\right)\right), & \Lambda_{\min }=1+\frac{p_{0}}{n}\left(2-s^{\prime}\right) & \text { if } n>1, \\
\Lambda_{\max }=1+\frac{p_{0}}{n+p_{0}}\left(s-\frac{1}{p}\right), & \Lambda_{\min }=1+p_{0}\left(1-\frac{1}{p}\right) & \text { if } n=1 .
\end{array}
$$

In both cases, $\Lambda_{\max }>\max \left(\hat{\lambda}_{1}, \Lambda_{\min }\right)$ if $s$ is sufficiently close to $1+1 / p$ so that we may choose $\lambda_{1}$ between these values to get (A) with $\sigma=1$. Moreover, $R \lambda_{1}<R \Lambda_{\max }<$ $p n /(n-(s-1) p)$ if $s$ is close to $1+1 / p$ since $p>p_{0}(n-1) /\left(n+p_{0}\right)$.

If $n \leq p_{0}(p-1)$ (i.e. $\left.p_{0} /\left(n+p_{0}\right) \geq 1 / p\right)$, put $\tilde{R}=p p_{0} /\left(p_{0}+1\right)$. Then $\tilde{R} \in[r, p)$ so that we may choose $R \in(\tilde{R}, p)$. Define $\Lambda_{\min }=\Lambda_{\min }(R)$ and $\Lambda_{\max }=\Lambda_{\max }(R)$ by (1). Then $\Lambda_{\max }(R)>\Lambda_{\min }(R)$ if and only if $R>p p_{0} /\left(p_{0}+p(s-1)\right)$. Since $\Lambda_{\max }(\tilde{R})=1+1 / p+$ $(s-1-1 / p) p_{0} /\left(n+p_{0}\right)>\hat{\lambda}_{1}$ for $s$ sufficiently close to $1+1 / p$, we have also $\Lambda_{\max }(R)>\hat{\lambda}_{1}$ for $s$ close to $1+1 / p$ and $R$ close to $\tilde{R}$. Consequently, $\Lambda_{\max }(R)>\max \left(\hat{\lambda}_{1}, \Lambda_{\min }(R)\right)$ if $s$ is close to $1+1 / p, R$ is close to $\tilde{R}, R>p p_{0} /\left(p_{0}+p(s-1)\right)$, so that we may choose $\lambda_{1}$ between these values to get (A) with $\sigma=1$ (and $r$ or $\lambda$ replaced by $R$ or $\lambda_{1}$, respectively). Moreover, $R \lambda_{1}<R \Lambda_{\max }(R)<p n /(n-(s-1) p)$ if $s$ is close to $1+1 / p$ and $R$ is close to $\tilde{R}$ since $p>p_{0}(n-1) /\left(n+p_{0}\right)$.

Proof of the Theorem. Let us write $F=F_{f}+F_{g}$ where $F_{f}$ or $F_{g}$ represents the contribution of the function $f$ or $g$, respectively, i.e.

$$
\begin{aligned}
& F_{f}(t, u)=f(\cdot, t, u(\cdot), \nabla u(\cdot)), \\
& F_{g}(t, u)=(\sigma+A) \mathcal{R} g(\cdot, t, u(\cdot)),
\end{aligned}
$$

where $\sigma>0$ and the operator $\mathcal{R}$ is described in [1, Section 11]. Denote by $\hat{f}$ and $\hat{g}$ the Nemytskiu operators defined by

$$
\begin{aligned}
\hat{f}(t, u, v) & =f(\cdot, t, u(\cdot), v(\cdot)), \\
\hat{g}(t, u) & =g(\cdot, t, u(\cdot)) .
\end{aligned}
$$

Let $s, \lambda, r$ be from Lemma 2. Denoting by Tr and $i$ the trace operator and the imbedding, 
respectively, the operator $F_{g}$ can be written in the form (cf. [1, p. 258])

$$
\begin{aligned}
F_{g}(t, \cdot): W_{\mathcal{B}}^{s / 2} & =W_{p}^{s}\left(\Omega, \mathbb{R}^{N}\right) \stackrel{\operatorname{Tr}}{\rightarrow} W_{p}^{s-1 / p}\left(\partial \Omega, \mathbb{R}^{N}\right) \stackrel{i}{\rightarrow} L_{r \lambda}\left(\partial \Omega, \mathbb{R}^{N}\right) \\
& \stackrel{\hat{g}(t, \cdot)}{\longrightarrow} L_{r}\left(\partial \Omega, \mathbb{R}^{N}\right) \stackrel{i}{\rightarrow} W_{p}^{s^{\prime}-1-1 / p}\left(\partial \Omega, \mathbb{R}^{N}\right) \stackrel{(\sigma+A) \mathcal{R}}{\longrightarrow} W_{\mathcal{B}}^{s^{\prime} / 2-1}
\end{aligned}
$$

for some $s^{\prime} \in(s, 1+1 / p)$ (the imbeddings are guaranteed by the inequalities in Lemma 2 ). Hence, using Lemmas 1 and 2 we can estimate

$$
\begin{aligned}
\left\|F_{g}(t, u)\right\|_{W_{\mathcal{B}}^{s^{\prime} / 2-1}} & \leq C\|\hat{g}(t, u)\|_{L_{r}\left(\partial \Omega, \mathbb{R}^{N}\right)} \leq C\left(1+\|u\|_{L_{r \lambda}\left(\partial \Omega, \mathbb{R}^{N}\right)}^{\lambda}\right) \\
& \leq C\left(1+\|u\|_{1 / r \lambda, r \lambda}^{\lambda}\right) \leq C\left(1+\|u\|_{p_{0}}^{\lambda-\varepsilon}\|u\|_{s, p}^{\varepsilon}\right) \\
& \leq C c(t)^{\lambda-\varepsilon}\left(1+\|u\|_{W_{\mathcal{B}}^{s / 2}}^{\varepsilon}\right) .
\end{aligned}
$$

Similarly, if $s, \lambda_{0}, \lambda_{1}, r, R$ are the constants from Lemma 3 then the operator $F_{f}$ can be written as

$$
\begin{aligned}
F_{f}(t, \cdot): W_{\mathcal{B}}^{s / 2} & =W_{p}^{s}\left(\Omega, \mathbb{R}^{N}\right) \stackrel{i \times \nabla}{\longrightarrow} W_{p}^{s}\left(\Omega, \mathbb{R}^{N}\right) \times\left(W_{p}^{s-1}\left(\Omega, \mathbb{R}^{N}\right)\right)^{n} \\
& \stackrel{i}{\rightarrow} L_{r \lambda_{0}}\left(\Omega, \mathbb{R}^{N}\right) \times\left(L_{R \lambda_{1}}\left(\Omega, \mathbb{R}^{N}\right)\right)^{n} \stackrel{\hat{f}(t, \cdot \cdot \cdot)}{\longrightarrow} L_{r}\left(\Omega, \mathbb{R}^{N}\right) \stackrel{i}{\longrightarrow} W_{\mathcal{B}}^{s^{\prime} / 2-1}
\end{aligned}
$$

together with the corresponding estimate

$$
\left\|F_{g}(t, u)\right\|_{W_{\mathcal{B}}^{s^{\prime} / 2-1}} \leq C(t)\left(1+\|u\|_{W_{\mathcal{B}}^{s / 2}}^{\varepsilon}\right)
$$

Remark 2. If we assume $f \equiv 0$ and $\sup _{t \in[0, T)}\|u(t)\|_{L_{p_{0}}\left(\partial \Omega, \mathbb{R}^{N}\right)} \leq c(t)$ instead of $\sup _{t \in[0, T)}\|u(t)\|_{L_{p_{0}}\left(\Omega, \mathbb{R}^{N}\right)} \leq c(t)$ in our Theorem then we may repeat the considerations above with the corresponding estimate

$$
\begin{aligned}
\left\|F_{g}(t, u)\right\|_{W_{\mathcal{B}}^{s^{\prime} / 2-1}} & \leq C\left(1+\|u\|_{L_{r \lambda}\left(\partial \Omega, \mathbb{R}^{N}\right)}^{\lambda}\right) \\
& \leq C\left(1+\|u\|_{L_{p_{0}}\left(\partial \Omega, \mathbb{R}^{N}\right)}^{\lambda-\varepsilon}\|u\|_{W_{p}^{s-1 / p}\left(\partial \Omega, \mathbb{R}^{N}\right)}^{\varepsilon}\right) \\
& \leq C c(t)^{\lambda-\varepsilon}\left(1+\|u\|_{W_{\mathcal{B}}^{s / 2}}^{\varepsilon}\right)
\end{aligned}
$$

under the following hypothesis on $p, p_{0}$ and $\hat{\lambda}$ :

$$
p>\max \left(1, \frac{p_{0}(n-1)}{p_{0}+n-1}\right), \quad \hat{\lambda}<1+\frac{p_{0}}{n-1} .
$$

This corresponds to the results of J. Filo in [4].

ExAmple. Let $n=N=1, \Omega=(-1,1), f \equiv 0, g(x, t, u)=u^{\lambda}, \lambda>1$, let $u_{0}$ : $[-1,1] \rightarrow \mathbb{R}^{+}$be a smooth function, $u_{0}(-x)=u_{0}(x)$ for $x \in[-1,1], u_{0}^{\prime}(1)=u_{0}^{\lambda}(1)>0$ and let the first four derivatives of $u_{0}$ restricted to the interval $[0,1]$ be non-negative. Then [3] implies that the solution $u$ is non-negative, it blows up in a finite time $T=T\left(u_{0}\right)$ and 
choosing $p_{0} \geq 1$ we get

$$
\begin{aligned}
\frac{1}{p_{0}} \frac{d}{d t} \int_{0}^{1} u^{p_{0}}(x, t) d x & =\int_{0}^{1} u^{p_{0}-1} u_{t} d x=\int_{0}^{1} u^{p_{0}-1} u_{x x} d x \\
& =-\int_{0}^{1}\left(p_{0}-1\right) u^{p_{0}-2} u_{x}^{2} d x+\left.u^{p_{0}-1} u_{x}\right|_{x=0} ^{1} \\
& \leq u^{p_{0}+\lambda-1}(1, t) \leq\left(\frac{\lambda-1}{T-t}\right)^{\frac{p_{0}+\lambda-1}{2(\lambda-1)}}=C(T-t)^{-\frac{p_{0}+\lambda-1}{2(\lambda-1)}}
\end{aligned}
$$

where we have used the estimate (2.1) from [3]. Hence $\|u(t)\|_{p_{0}}$ stays bounded if $\frac{p_{0}+\lambda-1}{2(\lambda-1)}<$ 1, i.e. if $\lambda>1+p_{0}$. This shows that the condition $\hat{\lambda}<1+p_{0} / n$ in our Theorem is (except for the equality sign) optimal if $n=1$.

\section{References}

[1] H. Amann, Parabolic evolution equations and nonlinear boundary conditions, J. Differential Equations 72 (1988), 201-269.

[2] H. Amann, Global existence for semilinear parabolic systems, J. Reine Angew. Math. 360 (1985), 47-83.

[3] M. Fila and P. Quittner, The blow-up rate for the heat equation with a non-linear boundary condition, Math. Methods Appl. Sci. 14 (1991), 197-205.

[4] J. Filo, Uniform bounds for solutions of a degenerate diffusion equation with nonlinear boundary conditions, Comment. Math. Univ. Carolin. 30 (1989), 485-495. 